\title{
Assessment of Efficacy and Safety of Thread Lift in Mid Face
}

\author{
DALIA I. ABADA, M.B.B.Ch.**; IMAN H. EL-MAADAWY, M.D.**; ABEER A. HODEIB, M.D.** and \\ SAMEH ELGHAMRY, M.D.* \\ The Department of Plastic \& Reconstructive Surgery* and The Department of Dermatology \& Venereology**, \\ Faculty of Medicine, Tanta University, Egypt
}

\begin{abstract}
Background: Aging is a natural process which occurs with development of human age as the skin becomes thin, dry and loses elasticity mainly in the sun exposed areas. It is caused by intrinsic and extrinsic factors and is affected by genetic, environmental, hormonal and metabolic factors. A lot of methods are used to overcome aging and produce rejuvenation of skin including noninvasive, invasive and surgical. Surgical threads are polypropylene sutures which placed under skin and create inflammation and fibrosis around them and restore vitality of skin so they could be a safe and effective method for lifting.
\end{abstract}

Objective: The aim of this study was to assess the safety and efficacy of thread lift in mid face lifting.

Patients and Methods: The current study included 12 patients with mild to moderate laxity of skin of mid face. Local anesthesia was injected and threads were inserted under the skin with suitable number for the degree of skin laxity, differ from patient to another.

Results: There was improvement in the mid face of patients immediately after the procedure by the mechanical effect of the threads. Further improvement was observed in follow up after one month and three months with variable degrees. Mild temporary side effects like pain, edema and ecchymosis, had been reported.

Conclusion: Thread lift is a simple, safe and effective method, which produced a long-maintained effect compared with other methods of skin lifting.

Key Words: Midface - Thread lift.

\section{INTRODUCTION}

As the person ages, the scaffolding dermal facial skin, as well as the fat component are weakened. The affected areas generally include cheeks, eyebrows, mandibular area and neck. This is attributed to the fact that the connective tissue of the skin becomes thinner and the elastic fibers collapses [1]. The surgical facelift procedure to correct facial aging has been, so far, an elaborated and complicated procedure that takes a long time to recover from [2]. The introduction of absorbable barbed sutures produces a lifting action for facial aging [3-6]. This delivers predictable long-term results with less morbidity, less downtime and greater patient satisfaction [7]. It is a simple approach that allows a safe reduction of mild degree of aged face. Depending on the material that makes up the sutures and filling threads, they have been classified as non-absorbable and absorbable threads [8].

\section{PATIENTS AND METHODS}

This study is clinical pilot study. It included twelve patients with mild to moderate mid face laxity. They were selected from the attendances of the Outpatient Clinics of Dermatology and Venerology Department and Plastic and Reconstructive Surgery Department, Tanta University Hospitals. The study was accepted by Ethical Committee of the university.

\section{Inclusion criteria:}

Patients with mild to moderate laxity of mid face, evidenced by moderately deep folds who accepted to be included in this study, signed a written consent and continued till the end of the follow-up period.

\section{Exclusion criteria:}

- Pregnant and breast-feeding females.

- Patients with thin translucent skin with minimal subcutaneous fat.

- Morbid obese patients with tight facial skin.

- Patients with overabundance of skin, requiring surgical removal.

- Patients with any procoagulative or thrombophilic disorders. 
- Patients having any infectious diseases or chronic diseases such as chronic renal failure, hepatic insufficiency, hepatitis, cardiovascular disorders, diabetes mellitus, thyroid disorders and cancer.

- Patients with history of keloid formation.

All patients were subjected to complete medical history taking, general and dermatological examination for any associated skin diseases and for exclusion of any systemic disease, complete blood picture to exclude anemia, bleeding disorders and any systemic disease and clinical assessment of the mid face laxity according to Glogaue photoaging scale ( $\mathrm{I}=$ mild; minimal wrinkles, mild pigment and no keratosis, $\mathrm{II}=$ moderate; wrinkles in motion, visible brown spots and palpable keratosis, III= advanced; wrinkles at rest, obvious discoloration and visible keratosis and IV=severe; wrinkles throughout, no normal skin and yellow-grey skin color) [9].

Digital photographs were taken before the procedure, immediately after the procedure, one month and three months after the procedure. They include front, right lateral and left lateral views. Therapeutic regimen as multiple threads inserted in the mid face on both sides according to the surface area was done.

\section{Procedure of thread lift:}

Before the procedure, the skin was cleansed with cotton gauze soaked in $70 \%$ ethanol. The procedure was performed in minor surgery room in Outpatient Clinic of Dermatology and Venereology Department, Tanta University Hospitals in a well-ventilated area. Permanent markers were used to mark out the entry, exit points and the pass way of the needle. Local anesthesia with $2 \%$ lidocaine and 1:200,000 epinephrine was injected in each side of the face. The needle trocar was introduced through the skin and is advanced along the mapped pathway. The trocar was removed, and the suture was inserted under the skin. The protruding suture was slightly pulled backward. Gentle finger massage is done over the suture. Two sides of the face were compared for symmetry. The protruding suture was snipped with suture scissor. Topical antibiotic cream was applied.

\section{Post procedure instructions:}

All patients were instructed to avoid rubbing and friction of the face. The patients used an antiinflammatory, anti-edematous cream and analgesic three times daily for one week if pain is marked.
Follow-up for assessment the efficacy of the threads and demonstration of any side effects as ecchymosis, swelling, infection or migration of threads and treating them were reported after 1 and 3 months.

Degree of improvement of wrinkles was reported according to physician assessment regarding to the five point rating system $(0=$ no improvement $(0 \%), I=$ mild $(1-25 \%), I I=$ moderate $(26-50 \%)$, $\mathrm{III}=$ marked $(51-75 \%)$ and IV=excellent $(76-$ $100 \%)(10)$ and patients' satisfaction. Pain assessment was done regarding 5 -point pain scale $(0=$ no pain, $\mathrm{I}=$ mild, $\mathrm{II}=$ moderate, $\mathrm{III}=$ severe and $\mathrm{IV}=$ intolerable) [11].

\section{Statistical analysis:}

Data were fed to the computer and analyzed using IBM SPSS software package version 20.0. (Armonk, NY: IBM Corp). Qualitative data were described using number and percent. Quantitative data were described using range (minimum and maximum), mean, standard deviation and median. Significance of the obtained results was judged at the $5 \%$ level [12].

\section{RESULTS}

This study was carried out on 12 female patients suffering from mild to moderate mid face laxity. There were 7 patients $(58.33 \%)$ aged 50 years or more and 5 patients $(41.67 \%)$ aged $30-<50$ years with mean \pm SD $49.25 \pm 7.24$.

As regards the degree of mid face laxity, 7 patients $(58.33 \%)$ had mild laxity and 5 patients $(41.67 \%)$ had moderate laxity.

The mid face laxity was corrected by the absorbable bidirectional pulling cog threads $20 \mathrm{G} \mathrm{X}$ 100 (150) 2D (Made in Korea). The number of the threads inserted for each patient was different. Seven patients (58.33\%) inserted 2 threads in each side of the mid face, 3 patients $(25 \%)$ inserted 3 threads in each side and 2 patients $(16.67 \%)$ inserted 4 threads in each side with mean \pm SD $2.58 \pm 0.79$ (Table 1).

There were 4 patients $(33.33 \%)$ getting marked improvement and 6 patients $(50 \%)$ getting moderate improvement. All of them $(83.33 \%)$ were satisfied with their procedure. The remaining 2 patients $(16.67 \%)$ had mild response and they were not satisfied with their procedure (Table 2), Figs. (1-3). 
Table (1): Descriptive analysis of the studied patients according to number of the inserted threads.

\begin{tabular}{lcc}
\hline Number of inserted threads & No. $=12$ & $\%$ \\
\hline 2 threads for each side & 7 & 58.33 \\
3 threads for each side & 3 & 25 \\
4 threads for each side & 2 & 16.67 \\
Min.-max. & \multicolumn{2}{c}{$2-4$} \\
Mean \pm SD & \multicolumn{2}{c}{$2.58 \pm 0.79$} \\
\hline
\end{tabular}

Table (2): Descriptive analysis of the studied patients according to number of the inserted threads.

\begin{tabular}{lcl}
\hline & No.=12 & \multicolumn{1}{c}{$\%$} \\
\hline Degree of improvement: & & \\
Marked & 4 & 33.33 \\
Moderate & 6 & 50 \\
Mild & 2 & 16.67 \\
Patients opinion: & & \\
Satisfied & 10 & 83.33 \\
Not satisfied & 2 & 16.67 \\
\hline
\end{tabular}

(A)

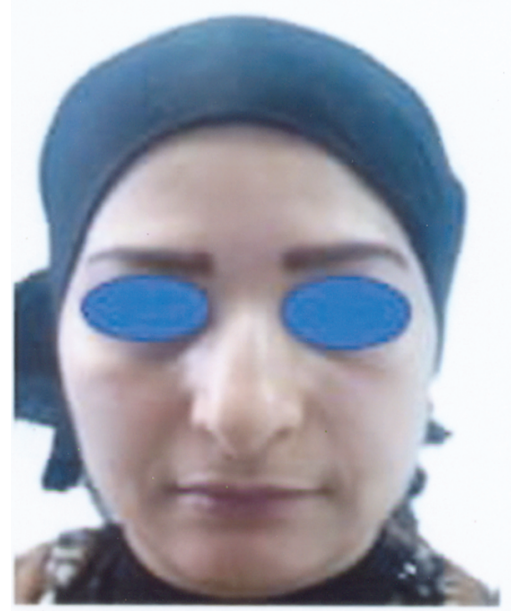

(B)

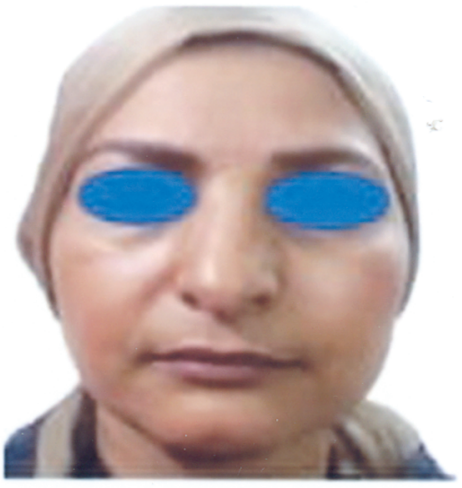

(C)

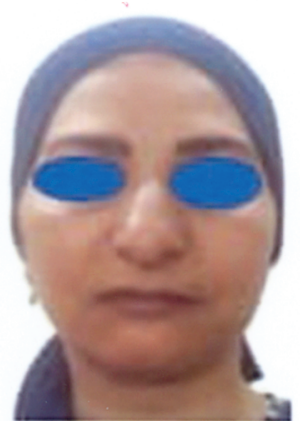

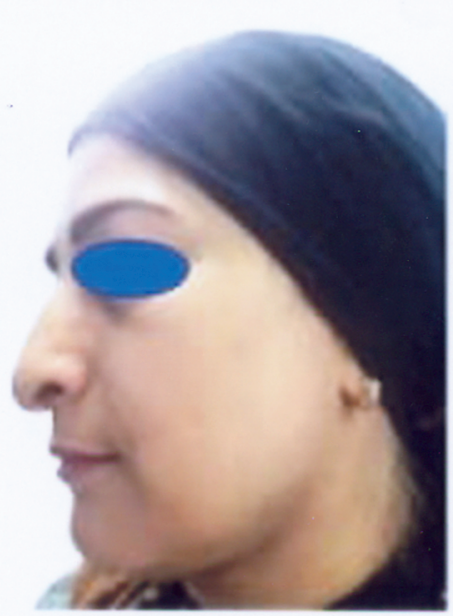
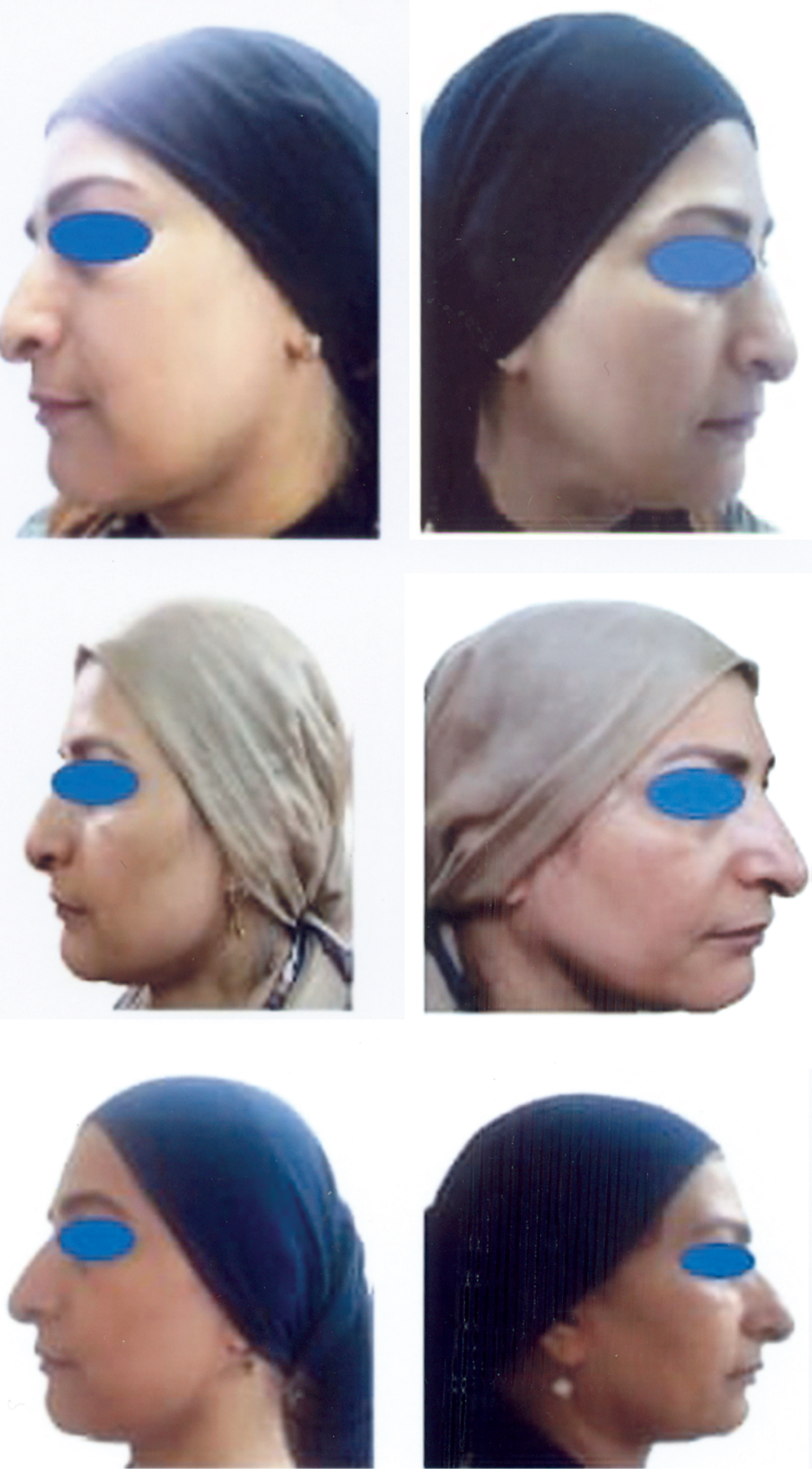

Fig. (1): 46yrs. old female patient with mild degree of mid face laxity; (A): Before the procedure, (B): 1 month after the procedure with moderate degree of improvement, (C): 3 months after the procedure with moderate degree of improvement. 
(A)

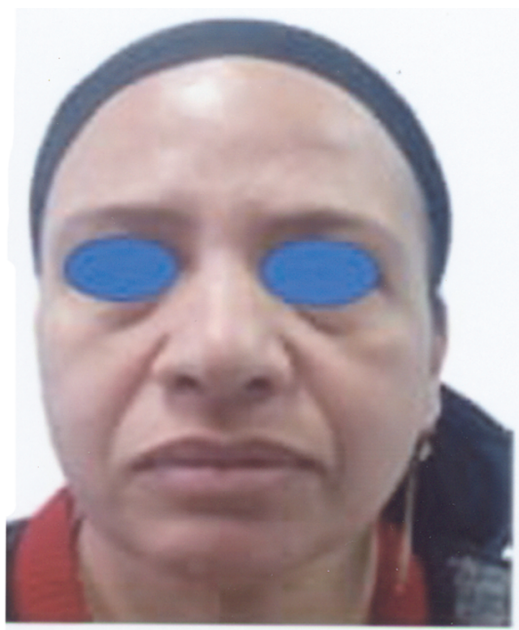

(B)

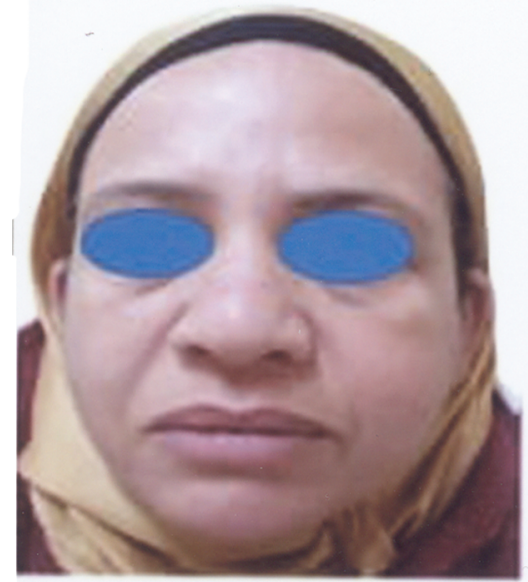

(C)

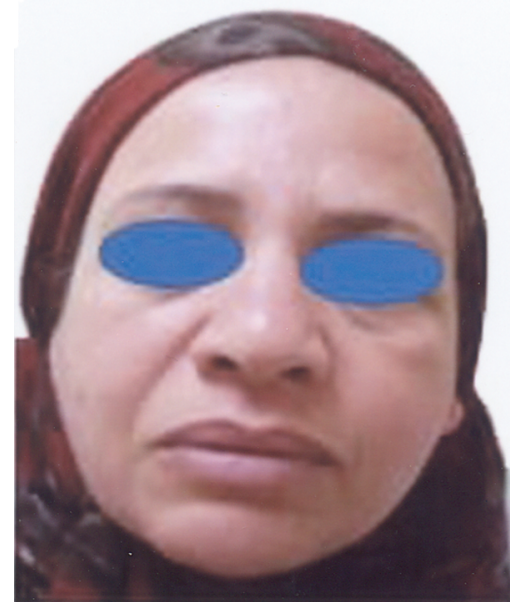

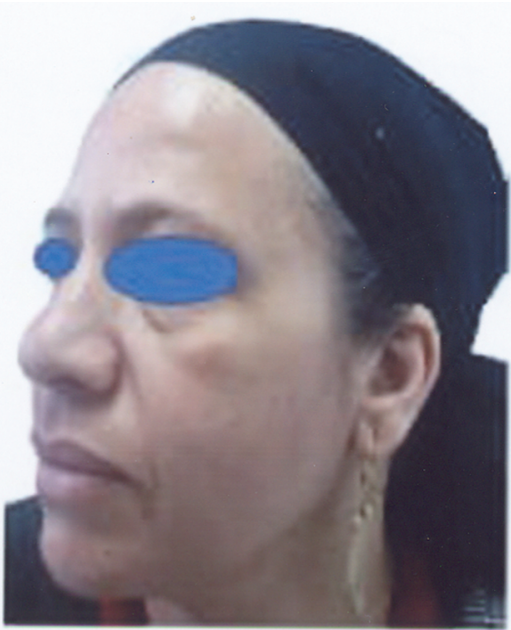
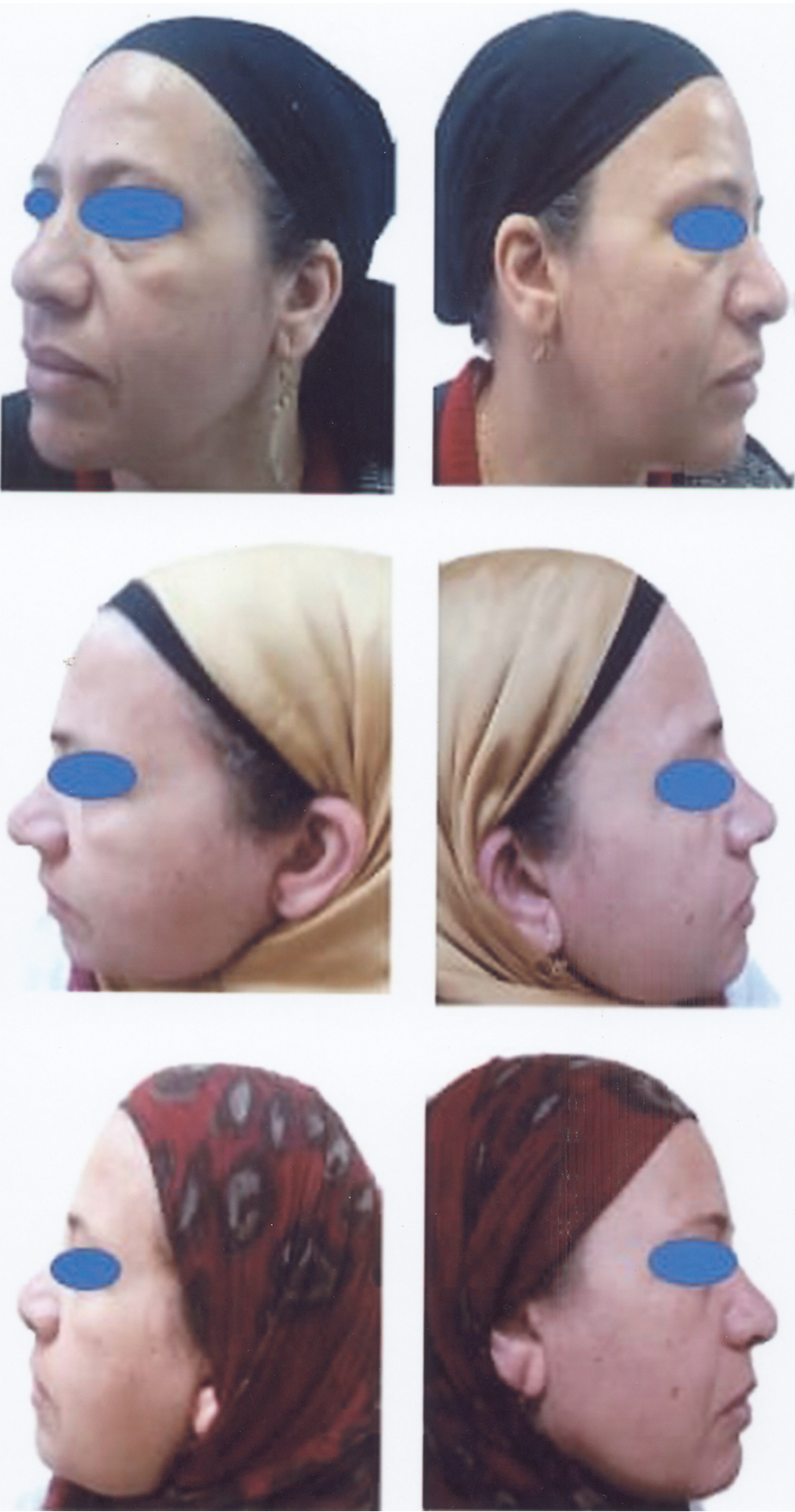

Fig. (2): 50yrs. old female patient with moderate degree of mid face laxity; (A): Before the procedure, (B): 1 month after the procedure with marked degree of improvement, $(\mathrm{C}): 3$ months after the procedure with marked degree of improvement. 
(A)

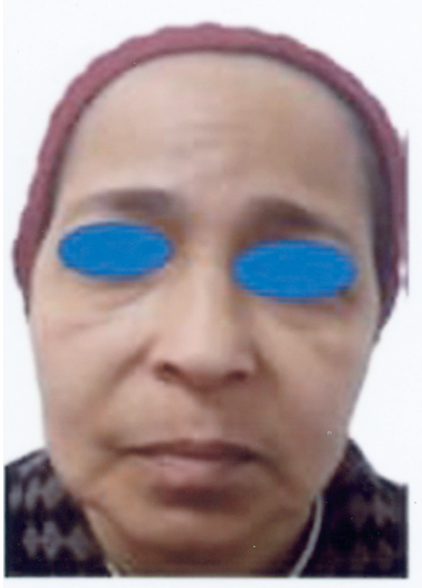

(B)

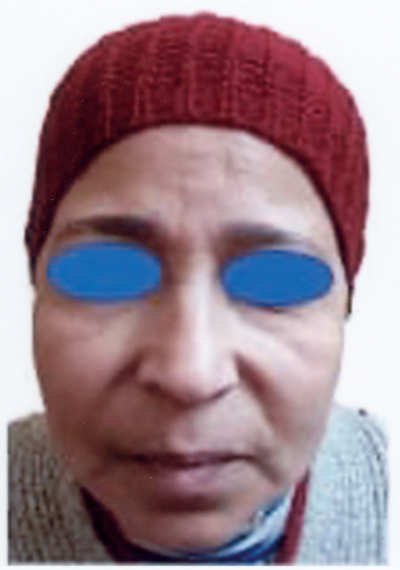

(C)

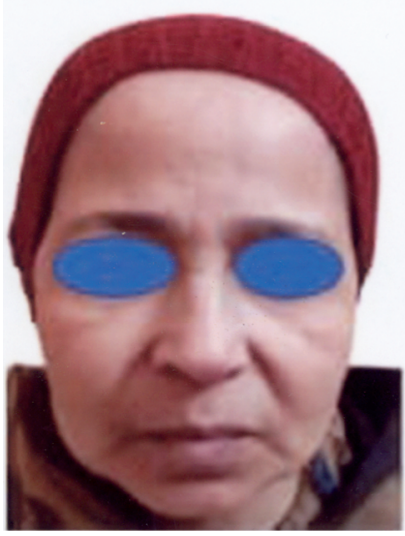

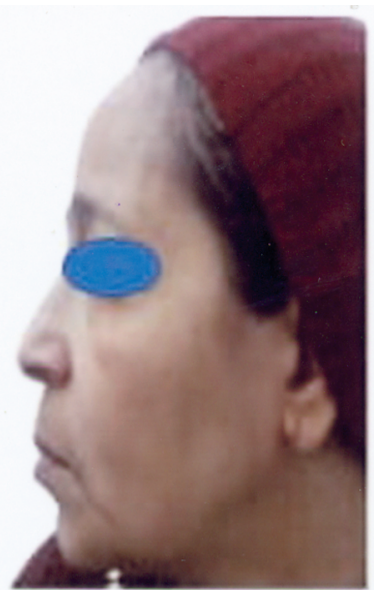
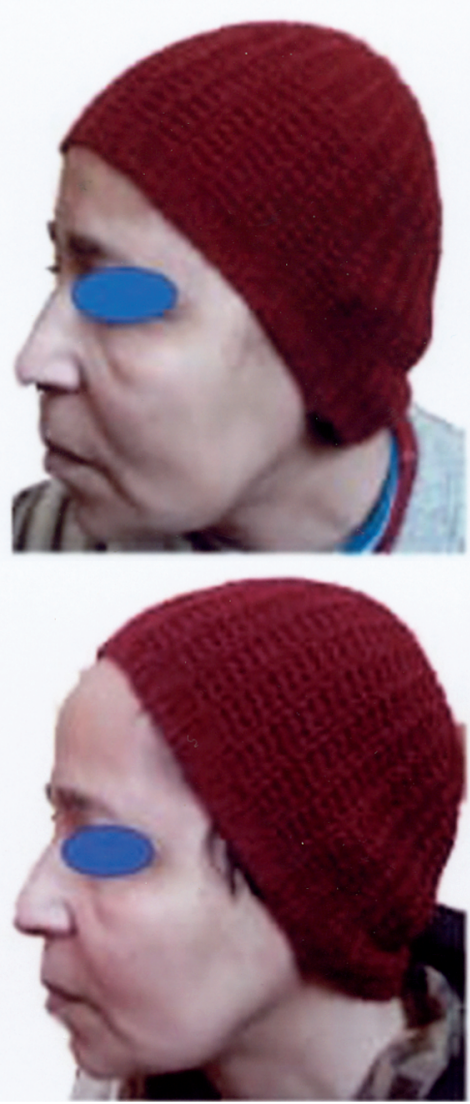
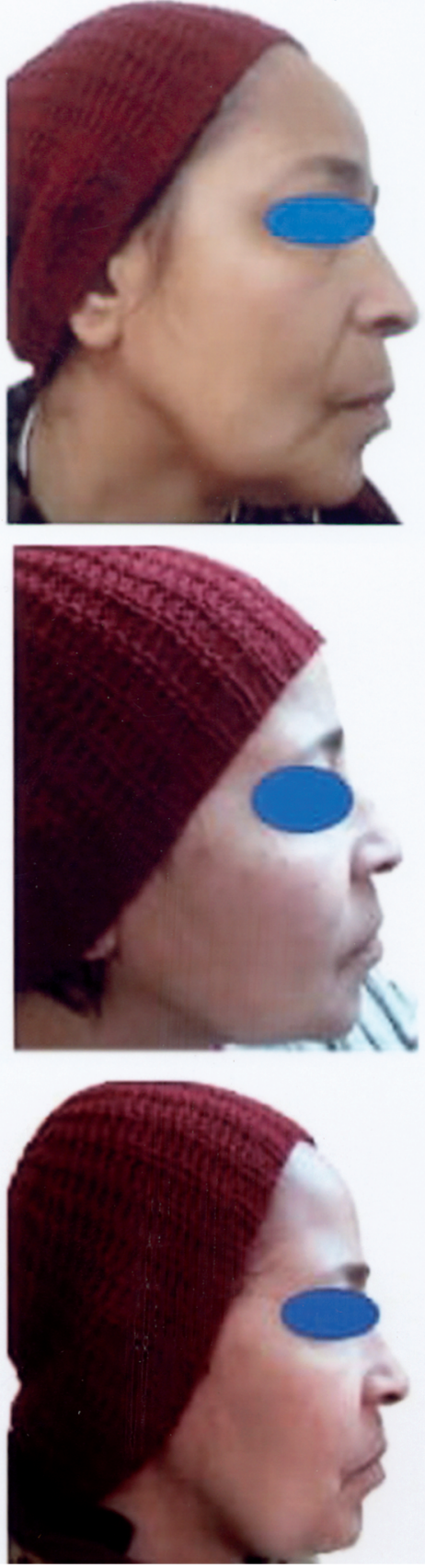

Fig. (3): 51 yrs. old female patient with moderate degree of mid face laxity; (A): Before the procedure, (B): 1 month after the procedure with mild degree of improvement, (C): 3 months after the procedure with mild degree of improvement.

Few side effects had been reported upon the procedure. All patients (100\%) had pain. Four patients $(33.33 \%)$ had slight ecchymosis and 3 patients $(25 \%)$ had slight edema.

Regarding 5-point pain scale, 6 patients (50\%) suffered from mild pain, 3 patients $(25 \%)$ suffered from moderate pain and 3 patients $(25 \%)$ suffered from severe pain. Pain disappeared after 7 days in 3 patients (25\%), after 10 days in 6 patients $(50 \%)$ and after 14 days in 3 patients (25\%). Ecchymosis disappeared after 7 days in one patient $(8.33 \%)$, after 10 days in one patient $(8.33 \%)$ and after 14 days in 2 patients (16.67\%). Edema disappeared after 7 days in one patient ( $8.33 \%$ ), after 10 days in one patient $(8.33 \%)$ and after 14 days in one patient $(8.33 \%)$ (Tables 3,4).

As regard follow-up of the patients after 1 month, 10 patients $(83.33 \%)$ had been improved with no side effects while after 3 months, they had more improvement while slight improvement with no side effects after 1 month reported in 2 patients $(16.67 \%)$ and still the same result after 3 months (Table 5). 
Table (3): Analysis of the reported side effects of the procedure.

\begin{tabular}{lll}
\hline Pain disappearance: & & \\
$\quad$ After 7 days & 3 & 25 \\
After 10 days & 6 & 20 \\
After 14 days & 3 & 25 \\
Ecchymosis disappearance: & & \\
After 7 days & 1 & 8.33 \\
After 10 days & 1 & 8.33 \\
After 14 days & 2 & 16.67 \\
Edema disappearance: & & \\
After 7 days & 1 & 8.33 \\
After 10 days & 1 & 8.33 \\
After 14 days & 1 & 8.33 \\
\hline
\end{tabular}

Table (4): Distribution of the studied patients according to degree of pain.

\begin{tabular}{lcc}
\hline Degree of pain & No. $=12$ & $\%$ \\
\hline Mild & 6 & 50 \\
Moderate & 3 & 25 \\
Sever & 3 & 25 \\
\hline
\end{tabular}

Table (5): Distribution of the studied patients according to follow-up after 1 and 3 months.

\begin{tabular}{lll}
\hline Follow-up & No.=12 & $\%$ \\
\hline After 1 month: & 10 & 83.33 \\
Improvement with no side effects & 2 & 16.67 \\
Slight Improvement with no side effects & & \\
After 3 months: & 10 & 83.33 \\
$\quad$ More improvement with no side effects & 10 & 16.67 \\
Slight improvement with no side effects & 2 & \\
\hline
\end{tabular}

\section{DISCUSSION}

With age, the facial support structure weakens and the facial fat has to be reduced. The affected areas generally include the cheeks, eyebrows, other areas around the eyes, the jowls and the neck. As the skin ages, the connective tissue in the skin becomes thinner and the elastic fibers undergo breakdown, so the face loses some of its elasticity and it begins to sag and wrinkle [13].

Rejuvenation is introduced by the preferred four (Rs) techniques, which are relaxation, refilling, reposition and resurfacing. Relaxation using BTX. Refilling using fat transfer or fillers. Reposition of sagging soft tissue can be achieved with various kinds of threads that have been improved with the development of biomaterials. Resurfacing procedures using mechanical force, chemical materials or lasers can rejuvenate fine wrinkles [14].

Thread lift may be a good alternative to the more invasive procedures that are necessary to correct problems of faces of elderly. Thread lift emerged because many people would like a face lift but cannot afford it or refuse the long recovery time of the standard face lift. Thread lifts cost less and require less downtime for many people. Some surgeons promote the thread lift as a lunchtime lift or weekend face lift [15].

Barbed sutures are modified polypropylene sutures which are either bidirectional or unidirectional. They intend to create fibrosis for longer lasting results [16]. Fibrous capsular contraction elicited around the threads. Barbs are the smallest part of the thread and likely to dissolve first [17].

The aim of this study was to evaluate the safety and the efficacy of thread lift in mild to moderate mid face laxity.

This current study was done on 12 female patients. The age of the patients ranged from 4060 years. Savoia et al., 2014 [18] reported the age ranged from 37-65 years in their study while Kalra, 2008 [15] reported that the ideal age for using thread lift ranged from 35-45 years.

In the current study, all patients were female for cosmetic complaints and this goes with Lycka et al., 2004 [19] and Han et al., 2016 [20]. They reported that all their studied patients were females.

This current study was done on the mid face. Studies done on thread lift are variable. Han et al., 2016 [20] did their study on mid face, while Badin et al., 2005 [21] did their study on mid and lower face. Kaminer et al., 2008 [22] and Savoia et al., 2014 [18] tried their studies on the whole face and neck.

This current study was done using the pulling cog threads which are absorbable bidirectional threads fixed inside trocars. The procedure did not require general anesthesia, no bleeding, no scarring and the patient could return to his daily activities shortly after the procedure. The effect of these threads produced by immediate mechanical effect then inflammatory reaction and fibrosis around threads and barbs on both sides in the bidirectional type but in the unidirectional type the barbs located on one side and the lift in one direction. Suh et al., 2015 [23] used the polydioxanone bidirectional cog threads. Atiyeh et al., 2010 [24] used the lunch time bidirectional threads which are a type of polypropylene barbed sutures. Sapountzis et al., 2014 [25] used new polypropylene barbed threads sealed with distal mesh named REEBORN which consist of five parts from distal to proximal as follow: A smooth part used as a guidance for the insertion of the needle, a segment with cogs, mesh segment, 
proximal cogs segment and smooth segment for fixation to the mesh and the deep temporal fascia. The needle, trocar and canula were used to create a sub SMAS plane while thread placement and the mesh were used for thread fixation to the deep temporal fascia. Vestita et al., 2016 [26] achieved facial rejuvenation by injection of substances aimed at stimulating collagen production called home made bio stimulating thread lift which consists of polydioxanone suture, a $1 \mathrm{~mm}$ lipofilling cannula and a small piece of polystyrene. Kang et al., 2017 [27] made a vertical lifting technique with polydioxanone sutures which inserted vertically downward in the anterior malar and submalar areas.

In this study, the number of the used threads was 2-4 threads, that were inserted in each side of the mid face for each patient according to the degree of laxity. Sapountzis et al., 2014 [25] used two threads for mid face, while Suh et al., 2015 [23] and Silva-Siwady et al., 2005 [28] tried 5 threads on each side for each patient, 3 for upper and central cheek and 2 for mandibular area.

In the current study, $83.33 \%$ of patients showed improvement and patients were satisfied with the used cog threads. Suh et al., 2015 [23] reported $87 \%$ success with cog threads used for 31 patients. Park et al., 2014 [29] reported 98\% success with cog threads used for 200 patients, while Kang et al., 2017 [27] reported $89.7 \%$ success with polydioxanone threads used for 39 patients.

Regarding the side effects of the procedure in the current study, the patients suffered from slight side effects as pain (100\%), slight edema (100\%) and slight ecchymosis (100\%) which resolved spontaneously. Other studies reported the same side effects $[18,19,21,23]$. More dangerous side effects were reported in Silva-Siwady et al., 2005 [28] as thread migration and partial expulsion. Rachel et al., 2010 [7] and Sulamanidze et al., 2011 [31] reported skin dimpling, hypercorrection, hypocorrection, transient paresthesia, thread migration and injury to major vessels, nerve branches, parotid capsule and duct. Kalra, 2008 [15] reported thread knots, infection and asymmetry. Yeo et al., 2017 [32] reported alopecia at 3 weeks after thread lifting then resolved at 5 months due to ischemia of tension anchoring process.

This current study approved that thread lift as an antiaging procedure is simple, effective with minimal invasive technique for mild and moderate laxity of the face with little spontaneously resolved side effects. This agrees with other studies [18-

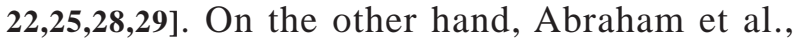

2009 [33] reported a lot of complications and they also found that the thread lift was unable to accomplish the goal of long term results with less morbidity, less downtime and greater patient satisfaction probably because it needs special experience and detailed knowledge of anatomy and the possibility of aberrant or different pathway of some nerves or vessels in some individuals. Also, the appropriate good selection of patients with mild or moderate face laxity and not to patients with marked laxity and cutaneous hypotonicity.

Some studies encourage the use of thread lift in combination with other procedure for better results as $\mathrm{Wu}, 2014$ [17] who made thread lift with botox in the glabella, brow and lateral orbital regions. Augmented effect of both as thread lift induced tightness and firmness while botox relaxed overactive contracting muscles and improved wrinkles. Moreover, hyaluronic acid as a filler with its viscoelastic properties and high-water retention capacity fills out aging hollows of the face. Williams and Smith, 2007 [34] preferred to do thread lift in combination with lipotransfer as liposuction utilized autologous fat as a natural filler for better results of rejuvenation procedure.

\section{REFERENCES}

1- Helfrich Y.R., Sachs D.L. and Voorhees J.J.: Overview of skin aging and photoaging. Dermatol. Nurs., 20 (3): 177-83, 2008.

2- Chaffoo R.A.: Complications in facelift surgery: Avoidance and management. Facial Plast. Surg. Clin. North Am., 21 (4): 551-8, 2013

3- Lee S. and Isse N.: Barbed polypropylene sutures for midface elevation: Early results. Arch. Facial Plast. Surg., 7 (1): 55-61, 2005.

4- Ruff G.: Technique and uses for absorbable barbed sutures. Aesthet. Surg. J., 26 (5): 620-8, 2006.

5- Sulamanidze M.A., Paikidze T.G., Sulamanidze G.M. and Neigel J.M.: Facial lifting with „,,APTOS”, threads: Feather lift. Otolaryngol. Clin. North Am., 38 (5): 110917, 2005.

6- Horne D.F. and Kaminer M.S.: Reduction of face and neck laxity with anchored, barbed polypropylene sutures (Contour Threads). Skin. Therapy Lett., 11 (1): 5-7, 2006.

7- Rachel J.D., Lack E.B. and Larson B.: Incidence of complication and early recurrence in 29 patients after facial rejuvenation with barbed suture lifting. Dermatol. Surg., 36 (3): 348-54, 2010.

8- De Lorenzi C.L.: Barbed sutures, rationale and techniques. Aesthet. Surg. J., 26 (2): 223-9, 2006.

9- Li L., Xi W., Wei L., Yang L., Wen-Ying X., Li-Wen H., et al.: Analysis of factor related to female facial wrinkles: A survey of 1004 chinese women of Han nationality (J). Chin. J. Pract. Aeshet. Plast. Surg., 15 (3): 126-8, 2004. 
10- Munavalli G.S., Turley A., Silapunt S. and Biesman B.: Combining confluent and fractionally ablative modalities of a novel 2790nm YSGG laser for facial resurfacing. Lasers Surg. Med., 43 (4): 273-82, 2011.

11- Kozarev J. and Vizintin Z.: Novel laser therapy in treatment of onchomycosis. J. Laser Health Acad., 1 (1): 1-8, 2010.

12- Corp I.B.M.: IBM SPSS statistics for windows, version 20.0. Armonk NY: IBM Corp: 2011

13- Khazanchi R., Aggarwal A. and Johar M.: Anatomy of aging face. Indian J. Plast. Surg., 40 (2): 223-9, 2007.

14- Kim B.J., Choi J.H. and Lee Y.: Development of facial rejuvenation procedures: Thirty years of clinical experience with face lifts. Arch. Plast. Surg., 42 (5): 521-31, 2015.

15- Kalra R.: Use of barbed threads in facial rejuvenation. Indian J. Plast. Surg., 41 (Suppl): S93-S100, 2008.

16- Wu W.T.: Barbed sutures in facial rejuvenation. Aesthet. Surg. J., 24 (6): 582-7, 2004.

17- Wu W.T.L.: Commentary on facial rejuvenation with fine barbed threads: The simple MIZ-lift. Aesthet. Plast. Surg., 38 (1): 75-7, 2014

18- Savoia A., Accardo C., Vannini F., Di Pasquale B. and Baldi A.: Outcomes in thread lift for facial rejuvenation: A study performed with happy lift ${ }^{\mathrm{TM}}$ revitalizing. Dermatol. Ther., 4 (1): 103-14, 2014.

19- Lycka B., Bazan C., Poletti E. and Treen B.: The emerging technique of the antiptosis subdermal suspension thread. Dermatol. Surg., 30 (1): 41-4, 2004.

20- Han S.E., Go J.Y., Pyon J.K. and Oh K.S.: A prospective evaluation of outcomes for midface rejuvenation with mesh suspension thread: "REEBORN lift". J. Cosmet. Dermatol., 15 (3): 254-9, 2016.

21- Badin A.Z., Forte M.R. and E. Silva O.L.: Scarless midand lower face lift. Aesthet. Surg. J., 25 (4): 340-7, 2005.

22- Kaminer M.S., Bogart M., Choi C. and Wee S.A.: Longterm efficacy of anchored barbed sutures in the face and neck. Dermatol. Surg., 34 (8): 1041-7, 2008.

23- Suh D.H., Jang H.W., Lee S.J., Lee W.S. and Ryu H.J.:
Outcomes of polydioxanone knotless thread lifting for facial rejuvenation. Dermatol. Surg., 41 (6): 720-5, 2015.

24- Atiyeh B.S., Dibo S.A., Costagliola M. and Hayek S.N.: Barbed sutures "lunch time" lifting: Evidence-based efficacy. J. Cosmet. Dermatol., 9 (2): 132-41, 2010.

25- Sapountzis S., Nikkhah D., Kim J.H. and Seo J.D.: Novel polypropylene barbed threads for midface lift-"REEBORN lifting”. Plast. Reconstr. Surg. Glob. Open, 2 (11): e 250, 2014.

26- Vestita M., Filoni A., Bonamonte D. and Giudice G.: The biostimulating thread lift. Plast. Reconstr. Surg. Glob. Open, 4 (9): e1015, 2016.

27- Kang S.H., Byun E.J. and Kim H.S.: Vertical lifting: A new optimal thread lifting technique for Asians. Dermatol. Surg., 43 (10): 1263-70, 2017.

28- Silva-Siwady J.G., Diaz-Garza C. and Ocampo-Candiani J.: A case of Aptos thread migration and partial expulsion. Dermatol. Surg., 31 (3): 356-8, 2005.

29- Park T.H., Seo S.W. and Whang K.W.: Facial rejuvenation with fine-barbed threads: The simple Miz lift. Aesthet. Plast. Surg., 38 (1): 69-74, 2014.

30- Sulamanidze M.A., Fournier P.F., Paikidze T.G. and Sulamanidze G.M.: Removal of facial soft tissue ptosis with special threads. Dermatol. Surg., 28 (5): 367-71, 2002.

31- Sulamanidze M., Sulamanidze G., Vozdvizhensky I. and Sulamanidze C.: Avoiding complications with Aptos sutures. Aesthet. Surg. J., 31 (8): 863-73, 2011.

32- Yeo S.H., Lee Y.B. and Han D.G.: Early complications from absorbable anchoring suture following thread lift for facial rejuvenation. Arch. Aesthet. Plast. Surg., 23 (1): 11-6, 2017.

33- Abraham R.F., De Fatta R.J. and Williams E.F. $3^{\text {rd: Thread- }}$ lift for facial rejuvenation: Assessment of long-term results. Arch. Facial Plast. Surg., 11 (3): 178-83, 2009.

34- Williams E.F. $3^{\text {rd }}$ and Smith S.P. Jr.: Minimally invasive midfacial rejuvenation: Combining thread lift and lipotransfer. Facial Plast. Surg. Clin. North Am., 15 (2): 209-19, 2007. 